\title{
Impaired ferritinophagy induced hepatic insulin resistance via endoplasmic reticulum stress of high fat diet mice
}

\author{
Chunjie Jiang ${ }^{1}$, Shanshan Zhang ${ }^{2}$, Hongmei Zeng ${ }^{1}$, Jingjing Liu ${ }^{1}$, Dan $\mathrm{Li}^{1}$, Li Chen ${ }^{1}$, Chao Gao ${ }^{3}$ \\ and Ping Yao \\ ${ }^{1}$ Huazhong University of Science and Technology, Wuhan, China, \\ ${ }^{2}$ Laboratory Animal Platform of Academy of Medical Sciences, Zhengzhou University, Zhengzhou, China and \\ ${ }^{3}$ National Institute for Nutrition and Health, Chinese Center for Disease Control and Prevention, Beijing, China
}

\section{Abstract}

Emerging evidence has been revealed that high fat diet (HFD) correlate with insulin resistance (IR) which could be induced by endoplasmic reticulum stress (ERS). Recently, obesity or HFD induced nonalcoholic fatty liver disease (NAFLD) could promote alteration of iron metabolism. Disorder of iron metabolism have been linked to unnormal metabolism of glucose and lipid. Herein, we investigated the effect of impaired iron homeostasis on hepatic IR, focusing on ferritinophagy. Male C57/6 J mice were administered with HFD (60\% energy from fat) or LFD (10\% energy from fat) for 10 weeks $(n=10)$, and Palmitic acid (PA)-insulin treated HepG2 cells were also established. Hepatic IR as evidenced by increased hepatic steatosis and decreased of $\mathrm{p}-\mathrm{AKT}(48 \%, \mathrm{p}<0.0005)$, p-GSK-3 $\beta(34 \%, p<0.05)$ in the liver of HFD mice. In addition, decreased iron level and expression NCOA4, as well as increased up-regulation of IRE1 $\alpha$ and EIF2 $\alpha$ were observed in HFD liver. By using desferrioxamine (DFO) and ferric ammonium citrate (FAC), we examined iron level on IRE1 $\alpha$ and EIF2 $\alpha$. And glucose uptake assay shown that FAC supplementation, and ERS inhibitors of 4-PBA and STF could improve the glucose uptake of HepG2 cells in the presence of PA. Furthermore, we evaluated the glucose uptake of HepG2 cells incubated with adenovirus which mediated overexpression of NCOA4, FAC, 4-PBA (ERS inhibitor) or STF (IRE1 inhibitor). Taken together, deficiency of iron induced by impaired ferritinophagy induced hepatic IR, partly by aggravating hepatic ERS, especially IRE1 signal pathway in vivo and vitro. These findings provide evidence and new insight for therapeutic strategy of iron deficiency in NAFLD.

\section{Conflict of Interest}

There is no conflict of interest 